\title{
Neonatal Intestinal Obstruction: A 5 Year Experience in a Tertiary Hospital in Enugu, Nigeria
}

\author{
Chukwubuike Kevin Emeka ${ }^{1}{ }^{*}$, Odetunde Oluwatoyin Arinola ${ }^{1}$, Ekwochi Uchenna $^{2}$, \\ Iheji Chukwunonso Chigozie ${ }^{2}$, Eze Thaddeus Chikaodili ${ }^{1}$
}

${ }^{1}$ Department of Surgery, Enugu State University Teaching Hospital, Enugu, Nigeria

${ }^{2}$ Department of Pediatrics, Enugu State University Teaching Hospital, Enugu, Nigeria

Email address:

chukwubuikeonline@yahoo.com (C. K. Emeka)

${ }^{*}$ Corresponding author

\section{To cite this article:}

Chukwubuike Kevin Emeka, Odetunde Oluwatoyin Arinola, Ekwochi Uchenna, Iheji Chukwunonso Chigozie, Eze Thaddeus Chikaodili. Neonatal Intestinal Obstruction: A 5 Year Experience in a Tertiary Hospital in Enugu, Nigeria. Journal of Surgery.

Vol. 7, No. 5, 2019, pp. 138-142. doi: 10.11648/j.js.20190705.15

Received: July 31, 2019; Accepted: August 18, 2019; Published: September 2, 2019

\begin{abstract}
Background: Neonatal intestinal obstruction is one of the most common surgical emergencies in a newborn requiring the services of a pediatric surgeon. This study reports the etiology, sex incidence, age of presentation, management and outcome of neonatal intestinal obstruction in pediatric surgical unit of a tertiary hospital in Enugu, Nigeria. Methodology: This was a retrospective study of neonates who presented, at Enugu State University Teaching Hospital, with intestinal obstruction over a 5-year period. Result: Thirty five neonates were recruited into the study. There were $24(68.6 \%)$ males and $11(31.4 \%)$ females. Five patients (14.3\%) were delivered before term (preterm) while thirty patients $(85.7 \%)$ were delivered at term. Most of the patients (54.3\%) presented after 72 hours from onset of symptoms. Abdominal distension was the most common symptomatology recorded in our patients. Necrotizing enterocolitis was the most common etiology in our patients accounting for 17 neonates (48.6\%). This was followed by anorectal malformation $5(14.3 \%)$, obstructed hernia $4(11.4 \%)$, intestinal atresia 4 (11.4\%), Hirschsprung's disease $3(8.6 \%)$, meconium plug $2(5.7 \%)$. Colostomy formation was the most common surgical procedure performed. Twenty percent of our patients developed post-operative complication and mortality was $22.9 \%$. Conclusion: Necrotizing enterocolitis is the most common cause of neonatal intestinal obstruction in the current study. With the mortality of $22.9 \%$, the outcome in our patients is still poor and requires some improvements.
\end{abstract}

Keywords: Intestinal Obstruction, Neonates, Necrotizing Enterocolitis

\section{Introduction}

The neonatal period is defined as the first 28 days after birth. Neonatal intestinal obstruction (NIO) is one of the most common surgical emergencies in a newborn requiring prompt intervention [1]. It is a life threatening condition in the newborn, with attendant high mortality especially in resource constraint environment like ours [2]. Neonatal intestinal obstruction is a common reason for admission to neonatal intensive care unit [3]. The incidence of NIO is estimated to be 1 in 2000 live births [4]. Failure of a full term neonate to pass meconium within 24 hours of birth should raise a suspicion of intestinal obstruction [5]. Wide varieties of pathologies are responsible for NIO. These include mechanical causes such as intestinal atresia, stenosis, malrotation, Ladd bands. Non mechanical (functional) causes include Hirschsprung's disease and necrotizing enterocolitis. In most cases, the prominent features of presentation of NIO are abdominal distension, bilious vomiting and failure to pass meconium/stool [6]. The level of intestinal obstruction determines the sequence of appearance of the symptoms. Investigations necessary in a neonate that has intestinal obstruction will include plain abdominal $\mathrm{x}$ ray which may show double bubble sign of duodenal obstruction or soap bubble appearance of meconium ileus, amongst others. Ultrasound scan, including antenatal (maternal) ultrasound, may show dilated bowel loops. Maternal polyhydramnios may suggest upper bowel obstruction. Upper and lower 
gastrointestinal tract contrast studies may be required in some cases [7]. Management of NIO is multidisciplinary. There is need for joint collaboration between the obstetrician at the time of prenatal diagnosis, the pediatrician that accepts the newborn in the delivery room, and the pediatric surgeon, who will determine the treatment according to the etiology [7]. Early diagnosis, prompt diagnosis and appropriate treatment have improved the outcome of NIO. Late presentation or delayed diagnosis of NIO can result in aspiration, sepsis, bowel gangrene and perforation [8].

The purpose of this study was to analyze the etiology, sex incidence, age of presentation, management and outcome of neonatal intestinal obstruction in pediatric surgical unit of a tertiary hospital in Enugu, Nigeria.

\section{Material and Method}

\subsection{Methodology}

This was a retrospective study of neonates who presented consecutively with intestinal obstruction to the pediatric surgical unit of Enugu State University Teaching Hospital Enugu, South East Nigeria. All patients from birth to the age of 28 days were recruited into the study. The study period was for 5 years (January 2014 to December 2018). Enugu State University Teaching Hospital serves the whole of Enugu State with a population of about 3 million. The hospital also receives referrals from its neighboring states. Patients who had surgery at a peripheral hospital for the condition before being referred to us for reoperation were excluded from the study. The reason for excluding these patients was because the exact pathology and the management offered to these patients in the peripheral hospital before presenting to us could not be ascertained. Cases with incomplete records and cases older than 28 days were also excluded from the study. All the neonates enrolled for the study were placed on intravenous fluid and intravenous antibiotics. Nasogastric decompressions were done to avoid aspiration.

Data were extracted from the case notes, operation notes, operation registers, and admission-discharge records. The information extracted include the age, gender, weight, gestation, diagnosis, symptoms, duration of symptoms before presentation, time interval between presentation and intervention, treatment offered, complications of treatment, outcome and duration of hospital stay.

\subsection{Prerequisite for Diagnosis}

The neonates were reviewed and diagnoses made by the consultant pediatric surgeon. Clinical features, laboratory, radiological and, in some cases, histological examinations were required for diagnoses to be confirmed. Neonates who have absent anal opening had cross table lateral $\mathrm{x}$-ray to assist in classifying the anomaly into high or low. Neonates that have bilious vomiting, had abdominal $\mathrm{x}$ rays checking for dilated bowel loop, multiple air fluid levels, absent rectal gas. Neonates that have delayed/non passage of meconium required rectal biopsy for histological confirmation of Hirschsprung's disease. Laboratory investigations done included full blood count and serum electrolytes to ensure fitness for surgery.

Ethical approval was obtained from the Ethics and Research committee of Enugu State University Teaching Hospital.

Role of funding source: No funding was received for this study.

Statistical Package for Social Science (SPSS) version 23 was used for data entry and analysis. Data were expressed as percentages.

\section{Results}

\subsection{Demography}

Forty three neonatal intestinal obstruction cases were seen during the study period but only 35 cases had complete case records and formed the basis of this report. A total of 4375 neonates were admitted into the newborn unit during the study period, out of which intestinal obstruction accounted for $0.8 \%$. There were $24(68.6 \%)$ males and $11(31.4 \%)$ females, with a male to female ratio of $2: 1$. Five patients $(14.3 \%)$ were delivered before term (preterm) while thirty patients $(85.7 \%)$ were delivered at term. The mean weight of the neonates at presentation was 2.3 kilograms (range: $1.8-$ 4.0) while the mean age was 7.1 days (range: 1-23 days). Regarding the time interval from onset of symptom to presentation, nineteen patients $(54.3 \%)$ presented after 72 hours, thirteen patients $(37.1 \%)$ presented between 24 to 72 hours and three patients $(8.6 \%)$ presented within 24 hours. The mean time from presentation to treatment was 2.5 days (range: $1-5$ ). The mean duration of hospital stay was 7.8 days (range: $1-18$ days). Demography of the patients is shown in table 1 .

Table 1. Demographic Characteristics of the study population.

\begin{tabular}{lll}
\hline Categories & Number & Percentage \\
\hline Gender & & \\
Male & 24 & 68.6 \\
Female & 11 & 31.4 \\
Maturity & & \\
Preterm & 5 & 14.3 \\
Term & 30 & 85.7 \\
Time of presentation & & \\
After 72 hours & 19 & 54.3 \\
24 to 72 hours & 13 & 37.1 \\
Within 24 hours & 3 & 8.6 \\
\hline
\end{tabular}

\subsection{Clinical Features}

The patients presented with a wide range of symptoms which included abdominal distension, bilious vomiting, failure to pass meconium, absent anal opening and fever. Some of the patients had more than one symptom. The frequencies of occurrence of the symptoms are shown in Table 2. 
Table 2. The clinical presentation of the patients.

\begin{tabular}{lll}
\hline Presenting Symptoms & $\begin{array}{l}\text { Number of } \\
\text { Patients }\end{array}$ & $\begin{array}{l}\text { Percentage } \\
(\%)\end{array}$ \\
\hline Abdominal distension only & 22 & 62.9 \\
Failure to pass meconium/stool & 5 & 14.3 \\
Abdominal distension plus vomiting & 3 & 8.6 \\
Bilious vomiting & 2 & 5.7 \\
Absent anal opening & 2 & 5.7 \\
\hline
\end{tabular}

\subsection{Investigations}

The cross table lateral x-ray of the five neonates $(14.3 \%)$ that have absent anal opening showed high anomalies. Abdominal x-rays revealed air within the bowel wall (pneumatosis intestinalis) in 17 patients (48.6\%) and multiple air fluid levels in 12 patients (34.3\%). Barium enemas were done in 3 neonates $(8.3 \%)$ and the results revealed the presence of the transition zone in the sigmoid colon. The full blood count results were within normal range except for one patient. Five neonates $(14.3 \%)$ had deranged serum electrolytes that needed correction.

\subsection{Diagnosis}

Necrotizing enterocolitis was the most common etiology in our patients who had neonatal intestinal obstruction accounting for 17 cases (48.6\%). Anorectal malformation was the second most common which occurred in 5 cases (14.3\%). Others are shown in Table 3.

Table 3. Diagnosis of the neonates.

\begin{tabular}{lll}
\hline Diagnosis & Number of neonates & Percentage (\%) \\
\hline Necrotizing Enterocolitis & 17 & 48.6 \\
Anorectal Malformation & 5 & 14.3 \\
Obstructed hernia & 4 & 11.4 \\
Intestinal atresia & 4 & 11.4 \\
Hirschsprung's disease & 3 & 8.6 \\
Meconium plug & 2 & 5.7 \\
\hline
\end{tabular}

\subsection{Procedures Performed}

Eight patients $(22.9 \%)$ had colostomy, five patients $(14.3 \%)$ had bowel resection and anastomosis, three patients $(8.6 \%)$ had herniotomy/herniorraphy, two patients $(5.7 \%)$ had water soluble contrast enema for the relief of the bowel obstruction. For the seventeen patients that have necrotizing enterocolitis, ten of them $(28.5 \%)$ were treated nonoperatively while seven patients $(20 \%)$ had peritoneal drain insertion (Table 4).

Table 4. Procedures performed on the neonates.

\begin{tabular}{lll}
\hline Procedure performed & $\begin{array}{l}\text { Number of } \\
\text { Patients }\end{array}$ & $\begin{array}{l}\text { Percentage } \\
(\%)\end{array}$ \\
\hline Non operative treatment of NEC & 10 & 28.6 \\
Colostomy & 8 & 22.9 \\
Peritoneal drain insertion & 7 & 20 \\
Bowel resection and anastomosis & 5 & 14.3 \\
Herniotomy/Herniorraphy & 3 & 8.6 \\
Enema reduction of meconium plug & 2 & 5.7 \\
\hline
\end{tabular}

\subsection{Post-operative Complications}

Twenty eight neonates (80\%) did not develop any complications while seven neonates (20\%) developed complications. Three neonates $(8.5 \%)$ developed sepsis, two neonates $(5.7 \%)$ developed wound infection, one neonate $(2.9 \%)$ each developed aspiration pneumonitis and anastomotic leak.

\subsection{Outcome}

Twenty five neonates (71.4\%) did well and were discharged. Eight neonates $(22.9 \%)$ died while two neonates $(5.7 \%)$ were discharged against medical advice.

\section{Discussion}

Neonates belong to a unique group of population because they have different physiologic features from older children and adults. In pediatric hospitals, neonates with intestinal obstruction constitute one of the most common causes for surgical admissions and they pose a challenge to both the neonate and the pediatric surgeon especially in developing countries [6]. Diagnosis of NIO could be a diagnostic challenge and the causes range from congenital to acquired anomalies, mechanical to functional etiologies. Neonatal intestinal obstruction is common indication for surgery in the newborn.

In the current study, there was a male preponderance with a male to female ratio of $2: 1$. This finding is consistently observed in many other reports too $[3,9,10,11]$. Most of our patients $(85.7 \%)$ were delivered at term, $14.3 \%$ of the neonates were preterm babies. Mohammed et al and Ademuyiwa et al in their study of neonatal intestinal obstruction reported that $13.7 \%$ and $13.1 \%$ of their patients respectively, were preterm babies [5, 9]. However, Singh et al recorded that $28 \%$ of their neonates that have intestinal obstruction were delivered preterm [12]. The mean weight of our patients, which was $2.3 \mathrm{~kg}$, was similar to what Anjali et al reported in their study [10]. However, Osifo et al in their study had a mean weight of 3.2 kilograms [2]. The mean age of our patients at presentation is similar to the findings of Anjali et al but Talari recorded a mean age of 11 days in their series [13]. Most of our patients (54.3\%) presented after 72 hours of onset of symptoms. This may be explained by poverty, ignorance and absence of effective health insurance scheme. In this series, the most common symptom was abdominal distension. A study done in Aba, Nigeria, also recorded abdominal distension as the most common symptom [14].

Abdominal radiograph was the most common investigation done on the neonates. The abdominal radiographs were in the forms of plain $\mathrm{x}$ rays or in cases of diagnostic difficulties, contrast $\mathrm{x}$ rays. Other studies also reported abdominal radiograph as the most common investigation performed in neonates that have intestinal obstruction $[12,14]$.

In this series, necrotizing enterocolitis (NEC) was the most common cause of neonatal intestinal obstruction. NEC is a 
multifactorial disease process of the gastrointestinal tract of neonates (especially premature neonates) that results in inflammation and bacterial invasion of the bowel wall [15]. Klein et al in their study of 137 neonates that have intestinal obstruction reported NEC as the most common etiology [16]. Some previous studies have reported intestinal atresia [10, $12,17]$, while some studies recorded anorectal malformation $[2,3,5,7,9,11,18,19]$ as the most common cause of intestinal obstruction in neonates. These differences in findings are difficult to explain. However, in the current study, the high number of preterm babies (14.3\%) in the current series may explain our findings.

Surgery was performed in 23 neonates $(65.7 \%)$. Formation of colostomy was the most commonly performed procedure in our patients $(22.9 \%)$. Colostomy was done in neonates who had anorectal malformation and Hirschsprung's disease. Colostomy was also the most commonly performed procedure in other studies $[14,20]$. On the other hand, Singh et al recorded intestinal resection and anastomosis as the most performed procedure in their series [12]. This could be explained by the fact that $50 \%$ of their patients had intestinal atresia which is treated by resection and anastomosis. For neonates that had NEC, they were managed non-operatively. This non-operative treatment included bowel rest and decompression, antibiotics, correction of hematological/electrolyte imbalance, provision of respiratory and cardiovascular support. Peritoneal drain was inserted if bowel perforation, evidenced by pneumoperitoneum, occurs.

Our finding is not similar to earlier reports from Nigeria with regard to post-operative complication rate $[11,14]$. Our complication rate, in the current study, of $20 \%$ seems to be the average of the other studies. These differences in complication rates may be explained by differences in the etiological patterns of intestinal obstruction in the different studies. The mortality rate in our series was $22.9 \%$. Though, this is comparable to the outcomes reported in other published series from Nigeria $[9,11]$, it is much higher than the mortality rate of less than $15 \%$ reported in Europe [21]. The low mortality in developed countries could be explained by antenatal detection, early presentation, early diagnosis, advances in neonatal anaesthesia/surgery, and presence of appropriate personnel and facilities.

\section{Conclusion}

Unlike many studies that reported anorectal malformation as the most common cause of neonatal intestinal obstruction. In the current study, the most common cause of neonatal intestinal obstruction was necrotizing enterocolitis and the second most common was anorectal malformation. However, similar to other studies done in the sub region, late presentation and high mortality is evident in the current study. Early diagnosis, early intervention, meticulous resuscitation, dedicated staff and good neonatal intensive care facilities are crucial for improving outcome in neonates that have intestinal obstruction. For future studies, we recommend a prospective approach and the enrollment of larger number of cases that will avail better analysis and provide basis for critical comparison.

\section{References}

[1] Adejuyigbe O, Jeje EA, Owa J, Adeoba EA. Neonatal intestinal obstruction in Ife, Nigeria. Niger Med J. 1992; 22: 24-28.

[2] Osifo OD, Okolo JC. Neonatal Intestinal Obstruction in Benin, Nigeria. 2009; 6 (2): 98-101.

[3] Nasir GA, Ralma S, Kadim AH. Neonatal intestinal obstruction. Eastern Mediterranean Health Journal. 2000; 6 (1): 187-193.

[4] Juang D, Snyder CL. Neonatal Intestinal Obstruction. Surg Clin North Am. 2012; 92 (3): 685-711.

[5] Mustefa Mohammed, Tadesse Amezene, Moges Tamirat. Intestinal Obstruction in Early Neonatal Period: A 3-Year Review of Admitted Cases from a Tertiary Hospital in Ethiopia. Ethiopia J Health. 2017; 27 (4): 393-400.

[6] Ashrarur Rahman Mitul. Congenital Neonatal Intestinal Obstruction. J Neonatal Surg. 2016; 5 (4): 41.

[7] Manuel Gil Vargas, Mariana L Miguel-Sardaneta, Michelle Rosas-Tellez, Dayana Pereira Reyes. Neonatal Intestinal Obstruction Syndrome. Pediatric Annals. 2018; 47 (5): e220e225. doi: org/10.3928/19382359-20180425-02.

[8] Kimura K. Bilious vomiting in the new born. Rapid diagnosis of intestinal obstruction. Am FAM physician. 2000; 61 (9): 2791-8.

[9] Ademuyiwa AO, Sowande OA, Ijaduola TK, Adejuyigbe O. Determinants of Mortality in neonatal intestinal obstruction in Ile Ife, Nigeria. Afr J Paediatr Surg. 2009; 6 (1): 11-13. doi: 10.4103/0189-6725.48568.

[10] Anjali Verma, Kamal Nain Rattan, Ravi Yadav. Neonatal Intestinal Obstruction: A 15 Year Experience in a Tertiary Care Hospital. J Clin Diagn Res. 2016; 10 (2): SC10-SC13.

[11] Ameh EA, Chirdan LB. Neonatal Intestinal Ostruction in Zaria. East Afr Med J. 2000; 77 (9): 510-3.

[12] Vijay Singh, Manish Pathak. Congenital Neonatal Intestinal Obstruction: Retrospective Analysis at Tertiary Care Hospital. J Neonatal Surg. 2016; 5 (4): 49.

[13] Talari VK, Sipala SK. A Clinical Study of Neonatal Intestinal Obstruction. IOSR Journal of Dental and Medical Sciences. 2017; 16 (8): 8-14. doi: 10.9790/0853-1608030814.

[14] Samuel Chidi Ekpemo, Nneka Okoronkwo. Neonatal Intestinal Obtruction in Aba, Nigeria. European Journal of Clinical and Biochemical Sciences. 2018; 4 (6): 69-72. doi: 10.11648/j.ejcbs.20180406.11.

[15] Thompson AM, Bizzarro MJ. Necrotizing enterocolitis in newborns: pathogenesis, prevention and management. Drugs. 2008; $1227-38$.

[16] Klein MD, Coran AG, Wesley JR, Drongowski RA. Hirschsprung's disease in the newborn. J Pediatr Surg. 1984; 19 (4): 370-4. doi: 10. 1016/s0022-3468(84)80255-9.

[17] Lister J. Development of neonatal surgery 1955-1980. J R Coll Surg Edinb. 1980; 25 (5): 324-32. 
[18] Chirdan LB, Uba F, Pam SD. Intestinal atresia: Management problems in a developing country. Pediatr Surg Int. 2004; 20 (11-12): 834-7.

[19] Momoh JT. Pattern of neonatal intestinal obstruction in Zarianorthern Nigeria. East Afr Med J. 1982; 59: 819-23.

[20] Sowande OA, Ogundoyin OO, Adejuyigbe O. Pattern and factors affecting management outcome of neonatal emergency surgery in Ile Ife, Nigeria. Surgical Practice. 2007; 11 (2): 7175. doi.org/10.1111/j.1744-1633.2007.00341.x.

[21] Bustos LG, Orbea GC, Dominguez GO, Gallindo IA, Cano NI. Congenital anatomic gastrointestinal obstruction: prenatal diagnosis, morbidity and mortality. An Pediatr (Barc). 2006; $62(2): 134-9$. 